\title{
LA NATURALISATION ACTE OU VECTEUR D'INTÉGRATION ?
}

\section{Retour sur l'histoire du débat dans le premier $\mathrm{xx}^{\mathrm{e}}$ siècle}

\section{Claire Zalc}

Le Seuil | « Pouvoirs »

$2017 / 1 \mathrm{~N}^{\circ} 160$ | pages 47 à 60

ISSN 0152-0768

ISBN 9782021343892

Article disponible en ligne à l'adresse :

https://www.cairn.info/revue-pouvoirs-2017-1-page-47.htm

Distribution électronique Cairn.info pour Le Seuil.

(c) Le Seuil. Tous droits réservés pour tous pays.

La reproduction ou représentation de cet article, notamment par photocopie, n'est autorisée que dans les limites des conditions générales d'utilisation du site ou, le cas échéant, des conditions générales de la licence souscrite par votre établissement. Toute autre reproduction ou représentation, en tout ou partie, sous quelque forme et de quelque manière que ce soit, est interdite sauf accord préalable et écrit de l'éditeur, en dehors des cas prévus par la législation en vigueur en France. Il est précisé que son stockage dans une base de données est également interdit. 


\section{CLAIRE Z A L C}

\section{LA NATURALISATION \\ ACTE OU VECTEUR \\ D'INTÉGRATION? \\ RETOUR SUR L'HISTOIRE DU DÉBAT \\ DANS LE PREMIER XXe SIĖCLE}

fin de penser la naturalisation, les approches françaises de philosophie politique convoquent le plus souvent le terme d'intégration. Cette notion fait débat ${ }^{1}$. Elle appartient à la fois à la langue du savant - on la retrouve chez Durkheim à la fin du XIX ${ }^{e}$ siècle ${ }^{2}$ - et à celle du politique. Elle tient sa pertinence des frontières juridiques qui distinguent l'étranger du national depuis la Révolution française. Si la question de l'intégration se pose, c'est que les étrangers «ne sont pas traités comme des nationaux », ils sont privés du droit de vote, se voient interdits un grand nombre d'emplois, peuvent être expulsés du territoire ${ }^{3}$. En France, le terme s'est imposé dans les questions relatives à la naturalisation, jusqu'à revêtir une dimension proprement institutionnelle dans les années 1990 où il vient désigner à la fois des politiques publiques et des institutions, à l'instar du Haut Conseil à l'intégration mis en place par le gouvernement de Michel Rocard en 19994. La notion prend ainsi aujourd'hui une forme contractuelle pour tous les étrangers

1. Dominique Schnapper, Qu'est-ce que l'intégration?, Paris, Gallimard, 2007, p. 16.

2. Émile Durkheim, De la division du travail social, Paris, Alcan, 1893.

3. Gérard Noiriel, Immigration, antisémitisme et racisme en France (XIX ${ }^{e}-X X^{e}$ siècles), Paris, Fayard, 2007, p. 525.

4. Le Haut Conseil est dissous quatorze ans plus tard, à la suite de la production d'un rapport controversé sur la laïcité dans l'enseignement supérieur. Cf. Jean Baubérot, «Le Haut Conseil à l'intégration et le bonnet d'âne ostensible», Mediapart.fr, 6 août 2013. 
accédant au séjour en France: la loi du 7 mars 2016 met en place un «parcours personnalisé d'intégration républicaine » d'une durée de cinq ans, qui comprend une formation civique et linguistique et dont la première étape consiste en la signature d'un «contrat d'intégration républicaine». L'ensemble de ces mesures témoigne d'une conception de l'intégration dont le point d'aboutissement constitue l'acquisition de la nationalité. Pourtant, face à cette thèse dominante selon laquelle la naturalisation vient sanctionner le parcours d'un immigrant «intégré», d'autres défendent l'idée que la naturalisation, par elle-même, serait vectrice d'intégration en donnant accès à un ensemble de droits. La naturalisation représenterait même «le processus d'intégration sociale par excellence, puisqu'il assure l'égalité - ne serait-ce que formelle mais tout aussi nécessaire - entre les immigrés devenus citoyens et les natifs face à la loi ${ }^{5}$ ».

Ce débat oppose deux conceptions de la nationalité qui sont résumées, trivialement, au cours d'un échange sur un plateau de télévision ${ }^{6}$. Alors que l'actrice d'origine belge Virginie Efira est interrogée sur les raisons qui l'ont poussée à acquérir la nationalité française quelques semaines auparavant, elle répond: «J'ai demandé la nationalité française pour voter.» Face à elle, le journaliste Yann Barthès paraît interloqué: "Simplement pour voter?» «Ben oui, pour quoi d'autre?» lui répond-elle du tac au tac, provoquant l'hilarité du public. Le journaliste tente alors une alternative: «Le plaisir d'être français ? " Ah oui! concède-t-elle avec une moue dubitative. Il y a une joie. Mais enfin... la joie d'être belge est assez équivalente, vous savez. » Le débat, dans cet échange télévisuel, vire au comique. Derrière ce dialogue de sourds, des points de vue sédimentés dans l'histoire. D'un côté, considérer la naturalisation comme une «faveur», un droit qui se «mérite», voire même un plaisir. De l'autre, rappeler que la naturalisation octroie des droits et réduit les inégalités entre étrangers et Français. Un petit retour en arrière permet de comprendre la manière dont les enjeux se sont peu à peu posés en France.

DIAGNOSTIC OU PRONOSTIC?

RETOUR SUR LA LOI DE I 927

On a tendance à oublier ce temps, le premier $\mathrm{xx}^{\mathrm{e}}$ siècle, où l'immigration était non seulement encouragée, mais considérée comme utile et nécessaire

5. Mirna Safi, Le Devenir des immigrés en France. Barrières et inégalités, thèse de sociologie, EHESS, 2007, p. 243.

6. «Quotidien», TMC, 13 septembre 2016. 
par de nombreux milieux. Figure d'exception sur le continent européen, la France s'impose en effet précocement comme l'un des principaux pôles mondiaux de l'immigration. Les bouleversements géopolitiques consécutifs à la Grande Guerre provoquent l'accélération des transferts de population et des mouvements de réfugiés, arméniens, assyrochaldéens, russes, grecs, bulgares, turcs... Avec la fermeture des frontières américaines par les lois des quotas de 1921 et de 1924, la France devient une destination privilégiée par ces migrants. Pour la première fois depuis 1891, le recensement de 1926 compte un volume entier consacré aux étrangers. La population étrangère gagne près d'un million de personnes en cinq ans seulement: de 1921 à 1926, elle passe de 1532000 personnes à 2409000 . Au total, $6 \%$ de la population en France est alors de nationalité étrangère, proportion qui, si elle augmente encore en 1931 $(6,6 \%)$, ne sera ensuite atteinte qu'en 1975 !

La priorité, dans les années 1920, est celle d'une immigration de peuplement, solution préconisée par un mouvement populationniste florissant qui cherche à tout prix à lutter contre le spectre du «déclin démographique». Depuis la fin du XIX ${ }^{e}$ siècle, de puissantes organisations natalistes, à l'instar de l'Alliance nationale pour l'accroissement de la population française, s'activent à ce sujet, brandissant l'étendard patriotique. L’enjeu est bien aussi militaire: le service militaire, réservé aux Français depuis 1872, est obligatoire depuis 1905. Le privilège des étrangers qui en sont dispensés devient inacceptable alors que les tensions diplomatiques en Europe s'exacerbent. Le million et demi de morts français de la Grande Guerre aiguise les peurs face à la «crise angoissante de la natalité française ", comme l'explique à la Chambre le député André Mallarmé, rapporteur de la loi de 1927 sur la nationalité7. Et si naturaliser était la solution? Ne pourrait-on transformer l'immigration en machine à produire de nouveaux Français?

C'est ainsi qu'est présentée et défendue, devant les parlementaires, la loi sur la nationalité du 10 août 1927 par les populationnistes, qui la qualifient de "planche de salut », "remède véritablement efficace au mal terrible dont souffre le pays, à ce mal qui, comme un cancer, le ronge et sur lequel, trop souvent, nous fermons les yeux: je veux dire la dépopulation », précise le député radical Charles Lambert. La loi entreprend de faciliter l'accession à la nationalité française des étrangers afin que ce «réservoir d'hommes » devienne «un complément à nos ressources

7. Journal officiel, Chambre des députés, débats, séances du 31 mars et du 7 avril 1927, p. $1100-1110$ et p. $1212-1221$. 
nationales défaillantes». À cette fin, elle fait preuve d'un libéralisme inédit et jamais égalé par la suite: seuls trois ans de présence sur le sol français sont exigés pour pouvoir demander la naturalisation, au lieu des dix ans instaurés auparavant par la loi de 1889. Les procédures administratives sont simplifiées. Les enfants nés en France qui n'ont pas répudié la nationalité française deviennent d'office français à leur majorité.

Les femmes françaises qui épousent un étranger peuvent conserver leur nationalité, brisant la norme alors en vigueur selon laquelle elles adoptaient systématiquement la nationalité de leur époux. La loi permet aussi aux femmes françaises qui se sont mariées à un étranger avant la promulgation de la loi de "réintégrer» leur nationalité d'origine sur simple demande. Si certains la taxent de féministe, qu'on ne s'y trompe pas. La femme y reste cantonnée à un rôle reproducteur ou, plus préci50 sément, de productrice de petits nationaux. Comme l'explique Charles Lambert: "[Il s'agit d'un] progrès pour le féminisme, non pas dans le sens où les féministes les plus ardentes l'entendent, car nous ne donnons pas à la femme française un bulletin de vote, mais nous lui assurons un moyen d'exercer son influence sociale au profit de son pays et au profit de l'avenir de sa race, nous lui permettons de conserver à la France de petits Français. »

Dans les débats autour de la loi de 1927, on retrouve l'opposition décrite plus haut. Jusqu'alors, la naturalisation était censée sanctionner l'assimilation des étrangers, maître mot des questions relatives à l'immigration dans l'entre-deux-guerres. En pratique, l'administration française s'employait pour cela à vérifier le degré d'assimilation des postulants à la nationalité, utilisant des méthodes variables d'un endroit à l'autre, hésitant entre critères socio-économiques et ethniques, à la faveur de la banalisation d'une lecture raciale des populations. La réduction de la durée de présence exigée sur le territoire à trois ans inverse les termes du débat: la naturalisation ne vient plus confirmer l'assimilation mais doit, au contraire, la favoriser. Tout individu né en France d'un parent étranger peut acquérir automatiquement la nationalité française s’il la réclame avant ses 21 ans: ce sont les Français «par déclaration». Comme l'explique Charles Lambert: «25000 enfants d'étrangers fréquentent les écoles primaires de Paris. Leurs maîtres vous diront qu'ils s'assimilent d'une façon remarquable et que beaucoup d'entre eux occupent les premières places de leur classe»; ils «seront demain d'excellents Français ». La loi de 1927 passe ainsi de l'ère des «diagnostics» à celle des "pronostics », comme l'explique la direction du ministère de la Justice en charge de l'appliquer. Elle prend un pari sur l'avenir. 
Le pari est assorti d'une contrepartie: dans le même temps est instaurée la possibilité de déchoir de la nationalité. Elle vise ceux qui commettent des «actes contraires à la sûreté intérieure et extérieure de l’État français », qui se livrent à des actes «incompatibles avec la qualité de citoyen français et contraires aux intérêts de la France» ou qui se soustraient aux obligations militaires. Et ce, pendant les dix ans qui suivent l'acquisition de la qualité de Français. Femme et enfants des intéressés peuvent être affectés par la mesure. Le principe de la déchéance fait l'objet de vifs débats. C'est à cette occasion que le garde des Sceaux, Louis Barthou, martèle combien la naturalisation reste une «faveur que le gouvernement peut accorder ou refuser». Face à lui, une partie de la gauche s'insurge contre la possibilité «de reprendre d'une main ce que [l'on a] généreusement attribué de l'autre». L'amendement qui propose la suppression de la déchéance est rejeté à une écrasante majorité et la loi adoptée.

Ses conséquences sont immédiates: le volume annuel des naturalisations double l'année suivante et plus de 650000 personnes acquièrent la nationalité française par décret entre 1927 et 1940, auxquelles il faut adjoindre les 250000 enfants devenus français par déclaration et les presque 100000 réintégrés dans la nationalité française. Au total, on porte au crédit de la loi de 1927 près d'un million de nouveaux Français.

\section{LES PRATIQUES DE LA FAVEUR}

L'histoire des naturalisations en France fait l'objet de travaux conséquents et complets qui s'articulent sur plusieurs dimensions : politiques et étatiques d'une part, bureaucratiques et sociologiques d'autre part, marquées par les travaux de Patrick Weil et d'Alexis Spire ${ }^{8}$. Elle a également suscité des enquêtes anthropologiques et sociologiques portant par exemple sur les cérémonies de naturalisation instaurées en 1993 ou sur les transformations subjectives imposées par la procédure ${ }^{9}$. Un certain nombre de travaux plus quantitatifs tente de mesurer les conséquences

8. Patrick Weil, Qu'est-ce qu'un Français? Histoire de la nationalité française depuis la Révolution, Paris, Grasset, 2002; Alexis Spire, Étrangers à la carte. L'administration de l'immigration en France (1945-1975), Paris, Grasset, 2005.

9. Sarah Mazouz, La République et ses autres. Politiques de la discrimination et pratiques de naturalisation dans la France des années 2000, thèse de sociologie, EHEss, 2010; Abdellali Hajjat, Les Frontières de l' identité nationale». L'injonction à l'assimilation en France métropolitaine et coloniale, Paris, La Découverte, 2012; François Masure, Devenir français? Approche anthropologique de la naturalisation, Toulouse, Presses universitaires du Mirail, 2014. 
des acquisitions de nationalité sur les trajectoires sociales et professionnelles des migrants. La naturalisation semble avoir des incidences positives sur les positionnements des immigrés sur le marché du travail, par le biais de divers facteurs, comme la réduction des obstacles relatifs à l'accès à l'emploi, l'accroissement de la mobilité et la baisse des discriminations, en particulier lorsque les immigrants sont originaires d'un pays à faibles revenus ${ }^{10}$. En France, la probabilité d'occuper un emploi hautement qualifié est plus forte de 7 points pour les hommes immigrés naturalisés que pour leurs homologues non naturalisés ${ }^{11}$. Focaliser l'analyse de la naturalisation sur la notion d'intégration conduit néanmoins à oblitérer la dimension pragmatique de l'acte au profit d'une conception sentimentale et largement idéologique de la nationalité. Le débat sur les relations entre naturalisation et intégration reflète une manière politique 52 et quelque peu ethnocentrée d'aborder la question, qui présuppose l'infériorité sociale des étrangers tendant supposément à vouloir de manière inévitable obtenir la nationalité. Or comme Alexis Spire le rappelle, la naturalisation «doit également se comprendre au regard des intérêts qu'elle présente pour l'État qui l'octroie. [...] Seule instance à pouvoir tracer la frontière entre celui qui est français et celui qui ne l'est pas », la puissance publique bénéficie, ce faisant, d'un «monopole de la définition légitime du national $»^{12}$.

Une plongée dans les dossiers de naturalisation du premier $\mathrm{xx}^{\mathrm{e}}$ siècle permet d'observer combien les manières d'appréhender les qualités des naturalisables évoluent selon les périodes. Demander sa naturalisation engage une démarche conditionnée par un certain nombre de critères objectifs, requis par les textes de loi, et d'autres plus subjectifs. S'il est difficile de rendre compte des phénomènes d'auto-élimination des immigrants qui pensent ne pas pouvoir satisfaire aux conditions requises par les autorités, il est néanmoins possible de montrer combien les façons de se présenter tentent de répondre aux attentes, mouvantes, de l'administration française. Les manières de décliner les identités témoignent des stratégies discursives utilisées en vue de se conformer au portrait supposé du «bon naturalisé». Ainsi par exemple, s'il est de bon ton, pour les postulants à la nationalité française, de se revendiquer commerçants au début du $\mathrm{xx}^{\mathrm{e}}$ siècle, il vaut mieux, dans les années 1930, taire

10. OCDE, La Naturalisation: un passeport pour une meilleure intégration des immigrés?, Paris, Éditions OCDE, 2011.

11. Didier Fougère et Mirna Safi, "Naturalization and Employment of Immigrants in France (1968-1999)", International Journal of Manpower, vol. 30, p. 83-96.

12. Alexis Spire, Étrangers à la carte, op. cit., p. 323. 
son appartenance au monde de la boutique pour se dire «ouvriers ${ }^{13}$. Dans les années 1910, le statut de commerçant vaut plutôt comme marque de notabilité et signe de bonne insertion dans la société française. Lors des demandes de naturalisation, l'activité commerciale est utilisée comme un indicateur susceptible de rassurer sur le niveau de fortune du postulant et, en particulier, sur son éventuelle capacité à payer les droits de naturalisation. En marge des dossiers, les fonctionnaires du bureau du sceau, chargés d'examiner les dossiers, mentionnent ainsi que tel étranger «offre les droits». L'installation d'une boutique présume également du désir de «s'établir définitivement en France». Le dossier de Hermann Tautmann, autrichien, représentant d'une société de commerce hambourgeoise, est construit autour des éléments de fortune et de stabilité: «Il se trouve dans une belle situation de fortune », note le fonctionnaire du bureau du sceau au crayon sur la première page de sa demande, avant de préciser qu'il «justifie d'une résidence ininterrompue en France depuis 1889» et de proposer la naturalisation. Le statut d'indépendant joue comme faire-valoir de «l'assimilation», sans constituer un critère suffisant. La taille et la stabilité de l'entreprise sont également scrutées à la loupe, constituant des indicateurs de la fortune et du degré d'insertion locale des postulants. Or les critères d'évaluation se modifient nettement dans l'entre-deux-guerres. Selon les termes de la loi de 1927, il faut, comme on l'a vu, avoir résidé trois ans sur le territoire français pour être naturalisé, mais ce délai peut être ramené à un an pour ceux qui ont «rendu des services importants à la France, apporté des talents distingués, introduit soit une industrie, soit des inventions utiles », mais également avoir créé des établissements industriels ou agricoles. Dans la pratique, le critère de la profession est rarement pris en compte même si, comme le montre Patrick Weil, «être dans une profession rurale peut permettre d'échapper au veto qui s'applique à celui qui a cherché à s'exempter du service militaire; à l'inverse, être commerçant ou investi dans une profession financière peut valoir ajournement, si des mauvais renseignements sont fournis ${ }^{14} »$.

Ainsi, Brikline Febous, qui tient un commerce de fourrure à Paris, se déclare ouvrier dans sa demande de naturalisation déposée en 1928: "J'ai l'honneur de vous demander d'être naturalisé français. J'habite Paris depuis 1913 et ma femme depuis 1905. J'ai un enfant, un petit garçon,

13. Claire Zalc, «Élite de façade et mirages de l'indépendance: les petits entrepreneurs étrangers en France dans l'entre-deux-guerres ", Historical Reflections, vol. 36, n 3, 2010, p. 94-112.

14. Patrick Weil, Qu'est-ce qu'un Français?, op. cit., p. 92. 
et plus tard je serai heureux qu'il serve la France. Je suis un honnête ouvrier et vous aurez les meilleurs renseignements sur moi ${ }^{15}$.» Il mentionne également le sexe de son enfant: les pères de garçon ont alors nettement plus de chances d'obtenir leur naturalisation. Lors de l'instruction des demandes de naturalisation, les enjeux militaires jouent en effet un rôle primordial. Les demandes déposées par des hommes âgés de 18 à 29 ans sont examinées d'un œil favorable, l'incorporation potentielle des postulants à la naturalisation constituant un critère essentiel de l'instruction des dossiers de naturalisations ${ }^{16}$. Il est ainsi porté une attention bienveillante à la présence, sur la demande, d'enfants de sexe masculin en regard d'enfants de sexe féminin qui paraissent «inutiles » à la France ${ }^{17}$. La nécessité d'accroître la puissance militaire du pays en hommes devient impérieuse dans le contexte de tension de la fin des années 1930. En 1939, une circulaire du 13 avril enjoint d'encourager et d'accélérer toutes les demandes de naturalisation qui pourraient accroître les effectifs de l'armée. Les procédures sont allégées et les délais d'instruction raccourcis. Sur les chemises des dossiers traités dans ce cadre, il est inscrit «URGENT SERVICE MILITAIRE». En 1939-1940, on observe une hausse sensible des naturalisations de jeunes hommes au cours d'une vague d'acquisitions qui prend valeur de campagne de recrutement militaire. On le voit, un ensemble de critères, non nécessairement mentionnés dans les textes, fait figure de moyen pratique pour différencier les «bons» des «mauvais» immigrés.

L'examen des dossiers confirme que le postulant à la nationalité française dans les années 1930 a tout intérêt à dissimuler sa qualité de «commerçant». Les avis défavorables se multiplient à l'égard des artisans et commerçants, dans un contexte de suspicion vis-à-vis de ces professions qui, pour reprendre l'expression administrative alors en vigueur, et relevée maintes fois dans les dossiers, ne présentent «aucun intérêt du point de vue national».

La légitimation des décisions à travers la notion d'intérêt rend compte des formes du pouvoir exercé par les agents. L'intérêt est l'objet de calculs de rentabilité afin d'évaluer ce que les naturalisations rapportent à l'État qui les octroie. Axiome de la politique d'immigration, l'intérêt oriente les décisions des agents, qui sont fondées sur un ensemble de calculs

15. Archives nationales, BB11/12406X28.

16. Linda Guerry, Le Genre de l'immigration et de la naturalisation, Lyon, ENs Éditions, 2013, p. 224-231.

17. Cf. Claire Zalc, Nanette Jacomijn Snoep, Hélène Lafont-Couturier et Laure Blévis (dir.), 1931. Les étrangers an temps de l'Exposition coloniale, Paris, Gallimard, 2008. 
coûts / avantages censés déterminer s’il est profitable, ou non, d'accorder la nationalité française à telle ou telle catégorie d'étrangers ${ }^{18}$. Le pouvoir discrétionnaire, autrement dit la possibilité d'agir «dans le cadre légal, à l'intérieur de normes qui autorisent un large pouvoir d'appréciation ${ }^{19}$ », constitue à la fois la cause et la conséquence de la conception de la naturalisation comme "faveur». Les raisonnements obéissent à une rationalité en finalité, pour reprendre la terminologie wébérienne, mais les objectifs poursuivis se déclinent sur plusieurs tableaux qui évoluent selon les contextes temporels et géographiques. Alexis Spire distingue ainsi, pour l'après-1945, trois logiques qui sous-tendent la politique d'immigration telle qu'elle est exercée par les agents, la logique de police, la logique démographique et la logique de main-d'œuvre. À cette fin, un ensemble d'indices et d'indicateurs, souvent implicites, sont employés au cours de l'instruction des dossiers en vue de déterminer l'utilité de la naturalisation: nationalité, profession, structure familiale. Or les agents peuvent jouer de plusieurs manières de cet intérêt. Si le raisonnement très économique, fondé sur la comparaison coûts / avantages, explique la prise en compte de critères professionnels dans les procédures, l'intérêt national, censé surplomber toute considération individuelle voire même comptable, permet d'accroître encore les marges d'interprétation des agents. La notion d'intérêt national arme les décisions et encadre les pratiques des agents en venant légitimer leur pouvoir discrétionnaire qui s'exerce au nom des intérêts, supérieurs, de l'État.

Cette caractéristique de la naturalisation est affirmée dans l'arrêt du Conseil d'État du 30 mars 1984 selon lequel «la naturalisation constitue une faveur accordée par l'État français à un étranger ${ }^{20}$ ». La décision de naturaliser est «un acte unilatéral de souveraineté, même si, depuis le début des années 1980, le nombre de recours n’a cessé de croître », souligne Alexis Spire ${ }^{21}$. Ce n'est qu'après l'adoption de la loi du 24 août 1993 qu'il est fait obligation à l'administration de motiver les décisions défavorables prises en matière de naturalisation. Lors de ses entretiens avec des postulants à la naturalisation, Sarah Mazouz décrit la tension entre ces deux termes, faveur ou droit, autour de la notion de mérite qui vient, pour l'administration préfectorale, «justifier l'octroi

18. Abdelmalek Sayad, "Coûts" et "profits" de l'immigration. Les présupposés politiques d'un débat économique", Actes de la recherche en sciences sociales, vol. 61, 1986, p. 79-82.

19. Olivier Beaud, La Puissance de l'État, Paris, Puf, 1994, p. 474.

20. CE, 30 mars 1984, Ministre des Affaires sociales et de la Solidarité nationale c. M. Abecassis, $\mathrm{n}^{\circ} 40735$.

21. Étrangers à la carte, op. cit., p. 324. 
de la nationalité comme une faveur et non comme un droit», alors que les naturalisés se «réapproprient cette catégorie sur un mode leur permettant, au contraire, de concevoir a posteriori la naturalisation comme leur étant revenue de droit $»^{22}$.

À l'instar de Simona Cerutti qui montre que la condition d'étranger, dans le Turin du XvıII siècle, est le produit d'incertitudes, un retour vers l'histoire de la première moitié du $\mathrm{xx}^{\mathrm{e}}$ siècle contribue à mettre au jour les différentes acceptions, selon les institutions et les moments, des «bons naturalisables » ${ }^{23}$. La qualification $\mathrm{du}$ «bon Français» ne répond pas à une définition objectivable, mesurable par l'administration et qualifiable de manière univoque et unanime. La conception qui fait de l'acquisition de la nationalité française une «faveur» accordée à l'étranger permet des interprétations variables. Elle comporte également des conséquences. Pour faire le contrepoids au libéralisme de la loi de 1927, des dispositions du texte restreignent les droits des naturalisés, les rendant ainsi inéligibles pendant dix ans. C'est le revers de la médaille de l'ère des "pronostics». Dès lors, les naturalisés sont placés dans une zone intermédiaire, entre Français et étrangers, en stage temporaire de francité. À la faveur du contexte xénophobe des années 1930, la liste des incapacités s'accroît. Les naturalisés font figure de boucs émissaires de la crise économique, dans de virulentes campagnes de presse. Un cortège de mesures est progressivement adopté interdisant aux naturalisés de moins de dix ans l'accès à la fonction publique, aux professions judiciaires puis médicales. Les naturalisés deviennent des Français de seconde zone. En 1938, le gouvernement d'Édouard Daladier interdit de voter pendant les cinq années qui suivent le décret de naturalisation. Le spectre d'application des mesures de déchéance est élargi: toute condamnation d'au moins un an d'emprisonnement expose le naturalisé à la perte de la nationalité française. Les naturalisés sont progressivement mis au ban de la société, notamment dans la sphère professionnelle, par le gouvernement de Vichy. Selon la loi du 12 juillet 1940, seules les personnes nées de parents français peuvent accéder aux cabinets ministériels. Puis sont évincés de la fonction publique tous ceux qui ne possèdent pas la nationalité française «à titre originaire, comme étant nés de père français » (loi du 17 juillet 1940). Cette disposition est étendue aux vétérinaires

22. Sarah Mazouz, «"Mériter d'être Français": pensée d'État et expérience de naturalisation », in Choukri Hmed et Sylvain Laurens (dir.), L'Invention de l'immigration, Marseille, Agone, 2008, p. 133.

23. Simona Cerutti, Étrangers. Étude d'une condition d'incertitude dans une société d'Ancien Régime, Paris, Bayard, 2012. 
(loi du 12 novembre 1940), avocats (loi du 10 septembre 1940), architectes (loi du 31 décembre 1940). La loi du 3 avril 1941 relative à l'accès aux emplois dans les administrations publiques interdit à toute personne, si elle n'est pas française et née de père français, d'être employée dans les administrations de l'État, des départements, des communes et des établissements publics, et même d'exercer des fonctions de direction dans un service public industriel exploité en régie. Les naturalisés sont touchés par la mesure. Le nombre de recours déposés par des individus devant le Conseil d'État pour demander que leur soit accordée une dérogation, à titre individuel, face à ces mesures laisse apercevoir la masse des personnes concernées. Plus encore, le régime de Vichy enclenche un mouvement de dénaturalisations.

Le régime de Vichy remet frontalement en cause la loi sur la nationalité de 1927. Quelques jours après son arrivée au pouvoir, le maréchal Pétain, par le texte du 22 juillet 1940, ordonne la révision de l'ensemble des naturalisations depuis le 10 août 1927. Il s'agit de revenir, rétroactivement, sur toutes les acquisitions de nationalité des douze années précédentes. Régler ses comptes avec la République signifie, pour l'État français, démembrer la loi de 1927 en rejetant hors de la communauté nationale ceux jugés «indignes» d'être français. Une Commission de révision des naturalisations est mise sur pied pour l'occasion; elle abat un travail titanesque, ouvrant plusieurs centaines de milliers de dossiers pour statuer sur chacun des cas. À nouveau, le pouvoir discrétionnaire est en marche qui aboutit à plus de quinze mille retraits de nationalité ${ }^{24}$.

Cet épisode vient ici révéler, en creux, ce que représente la naturalisation non plus pour l'État qui l'accorde mais pour les individus. La publication de décrets de retraits de la nationalité française ne constitue pas une mesure simplement formelle. L'exclusion de la communauté nationale implique un ensemble de conséquences, matérielles, juridiques et sociales, qui modifient les conditions d'existence des dénaturalisés. Les caractéristiques complexes de la «qualité» de Français n’apparaissent finalement qu'à la lumière de sa perte. Le problème est d'abord légal: en perdant la nationalité française, les dénaturalisés se retrouvent dans la précarité du statut d'étranger, ce qui, pour les Juifs, conduit bien souvent à précipiter leurs arrestations.

24. Claire Zalc, Dénaturalisés. Les retraits de nationalité sous Vichy, Paris, Seuil, 2016. 
La dénaturalisation signifie aussi perdre un statut, au sens d'un ensemble de droits et d'obligations mais également de positions interrelationnelles, statut social envié, enviable et particulièrement précieux dans le contexte de la Seconde Guerre mondiale ${ }^{25}$. Redevenir étranger ne revient pas uniquement à se retrouver plongé dans des questions de papiers d'identité. La perte de la nationalité française conduit aussi certains à perdre leur emploi, ce qui vient rappeler le nombre des professions réservées aux nationaux. Michel Anastasi, 19 ans, dénaturalisé avec ses parents, son frère et sa sœur en mars 1942, travaillait à la Compagnie générale transatlantique. En conséquence de la décision, «il est licencié et son livret de travail retiré», se plaint sa mère dans sa supplique au maréchal Pétain ${ }^{26}$.

Perdre la nationalité s'accompagne d'un lot d'exclusions. Un administrateur de la Maison des gazés sollicite une audience le 23 mai 1941

58 auprès de Xavier Vallat, commissaire général aux questions juives, pour protester contre les conséquences des dénaturalisations sur les «anciens combattants israélites»: si on leur retire la nationalité française, ils ne pourront plus être membres de cette institution mise en place par la Fédération nationale des combattants volontaires au Mont-Dore, dans le Puy-de-Dôme, afin de proposer des cures aux asthmatiques et gazés de la Première Guerre mondiale ${ }^{27}$. La question de la perte des droits liée à la dénaturalisation fait débat. Elle soulève des questions de rétroactivité. Faut-il ainsi retirer la carte de combattant délivrée à des étrangers naturalisés ayant perdu la nationalité française en exécution de la loi du 22 juillet 1940 ? Le secrétaire d'État chargé des anciens combattants «estime qu'ils doivent perdre, en même temps, la qualité de combattant, puisque le bénéfice de celle-ci n'a jamais été pour eux de droit strict et que la condition de naturalisation (servant de base à la mesure bienveillante) a disparu ${ }^{28}$ ».

Les conséquences sont aussi symboliques, négation des services militaires accomplis, humiliation des notifications, remise des papiers d'identité au commissariat du domicile. Elles touchent également les proches des dénaturalisés. Le 22 janvier 1942, Raymond Gomez, dont les parents ont été dénaturalisés en mars 1941, adresse une lettre au directeur de cabinet du maréchal Pétain, Henry du Moulin de Labarthète: «Si cette décision a profondément affecté mes parents qui m’ont élevé

25. Étienne Anheim, Jean-Yves Grenier et Antoine Lilti, «Repenser les statuts sociaux», Annales. Histoire, sciences sociales, vol. 68, n 4, 2013, p. 950.

26. Lettre du 8 septembre 1942, Archives nationales, BB/11/11024, art. 27139X29.

27. Centre de documentation juive contemporaine, CIX-4, fonds CGQJ, France.

28. Archives du Conseil d'État, AL/4476, art. 231407. 
dans l'amour de la France, elle m'a atteint moi-même dans mes chères affections de fils affectueux et autant dans mes sentiments de Français, car si la mesure qui atteint mes parents ne me frappe pas matériellement elle me blesse profondément moralement. Aussi je me permets, M. le Directeur, de vous demander de faire reconsidérer la mesure qui vise mes parents ${ }^{29}$.»

La dépossession de la nationalité s'accompagne ainsi d'une perte de statut et d'une forme de mort sociale. Elle augmente substantiellement les risques d'être arrêtés, déportés et assassinés pour les Juifs redevenus étrangers. C'est à l'aune des griefs et des blessures endurés par les dénaturalisés qu'on réalise la portée des préjudices occasionnés par la perte du statut national.

Revenir sur le premier $\mathrm{xx}^{\mathrm{e}}$ siècle permet d'aborder autrement la question des relations entre naturalisation et intégration. L'association si fréquente, dans les débats, entre ces deux termes gagne à être historicisée: elle reflète d'abord le point de vue de pouvoirs publics soucieux d'accroître dans l'entre-deux-guerres le nombre de nationaux, dans un contexte de crise démographique, tout en prétendant garder la mainmise sur leur sélection. Dès lors, la notion d'intégration vient légitimer les processus, mouvants selon les périodes et les besoins, de détermination des «bons» et des «mauvais» naturalisables. Enjeu crucial de souveraineté, les naturalisations relèvent alors largement du pouvoir discrétionnaire du gouvernement. Il s'agit d'une «faveur», clame le garde des Sceaux devant la Chambre des députés en 1927, terme qui va faire florès. Néanmoins, d'autres lectures sont possibles, notamment lorsqu'on privilégie une approche microsociale, à partir des dossiers de naturalisation. Cette matière archivistique émeut et fascine parce qu'elle met en scène le moment inaugural de la requête, dont les formes varient selon les périodes, qui initie le processus durant lequel se joue une "véritable opération de magie politico-sociale», pour reprendre les termes d'Abdelmalek Sayad, qui «a apparemment pour fonction de transformer en naturels d'un pays, d'une société, d'une nation, des individus qui ne le sont pas et qui demanderaient à l'être ${ }^{30}$. Et l'on y découvre alors

29. Lettre du 22 juillet 1942, Centre des archives contemporaines, 19770881 / 188, art. 33042X33.

30. Abdelmalek Sayad, "Naturels et naturalisés ", Actes de la recherche en sciences sociales, vol. 99, 1993, p. 26. 
ce que signifie, en pratique, la naturalisation, autrement dit l'accès à un statut aux multiples dimensions, juridiques bien entendu, mais également symboliques, politiques, économiques et sociales.

Naturalisation et intégration semblent bien souvent liées dans les débats politiques et philosophiques. Les uns défendent l'idée que la naturalisation vient sanctionner le parcours d'un immigrant "intégré", d'autres défendent la thèse selon laquelle la naturalisation, par elle-même, est vectrice d'intégration en donnant accès à un ensemble de droits. Ce débat oppose deux conceptions de la nationalité: d'un côté, une "faveur» qui se "mérite»; de l'autre, un droit réducteur d'inégalités. Cet article propose de revenir sur la mise en place des termes de ce débat dans la France du premier $X X^{e}$ siècle, pour discuter la pertinence de cette alliance sémantique et conceptuelle. Et s'il valait mieux considérer la nationalité comme un statut? 